\title{
Descompresiva microvascular en la neuralgia del trigémino: factores pronósticos
}

\author{
F. Alberione $* *, * * *, * * * * ;$ A. Arena**,***,**** y R. Matera*,**,***,**** \\ *Hospital Italiano. **Hospital Británico. ***Sanatorio Agote. ****Clínica Sagrada Familia. Buenos Aires. Argentina.
}

\section{Resumen}

Objetivo. Presentar nuestra experiencia de la descompresiva microvascular en la neuralgia del trigémino típica e identificar los factores pronóstico.

Material y métodos. Estudio retrospectivo de una serie de 89 casos intervenidos entre 1995 y 2005. Se analizaron las variables con significación pronóstica atendiendo a criterios demográficos, duración evolutiva de la neuralgia, cantidad de ramas afectadas, hallazgo quirúrgico y material utilizado en la descompresión. Los datos obtenidos fueron analizados por la prueba de $\chi^{2}$.

Resultados. La tasa de muy buena evolución al año fue del $77 \%$. De las variables analizadas no alcanzaron significación estadística la edad y antecedentes de HTA. Juegan un papel desfavorable: el sexo femenino, la duración evolutiva de la neuralgia mayor a 2 años, el compromiso de las tres ramas del trigémino, la compresión venosa y la utilización de músculo como material de separación del conflicto neuro-vascular.

Conclusión. La DMV es una técnica eficaz y segura. No recomendamos utilizar músculo como elemento de interposición vasculo-nerviosa debido a los malos resultados observados. Plantear otra modalidad terapéutica en los casos de afectación de las tres ramas trigeminales.

PALABRAS CLAVE: Neuralgia del trigémino. Descompresiva microvascular. Factores pronósticos. Seguimiento.

Microvascular descompression for trigeminal neuralgia: pronostic factors

\section{Summary}

Background. We describe our experience of the MVD in the typical trigeminal neuralgia and identify

Recibido: 11-01-08. Aceptado: 12-02-08 the prognostic factors.

Method. A retrospective studio of 89 cases between 1995-2005 was used. The prognostic significant data evaluated were demographics data; duration of neuralgia; the affected divisions involved; surgical findings; used material for the decompression. The data analysis was made with the $\chi^{2}$ test.

Results. We have found an excellent outcome in $77 \%$ one year later. The age and the antecedent of hypertension disease were not statistically significant. A poor outcome was observed for: female sex, neuralgia lasting longer than two years, the three divisions involved, venous compression and the muscle used as surgical material.

Conclusions. The MVD is an effective and reliable technique. The use of muscle is not recommended. When the three trigeminal divisions are involved we should choose another technique.

KEY WORDS: Trigeminal neuralgia. Microvascular decompression. Prognostic factors. Outcome.

Introducción

La neuralgia del trigémino (NT) típica o clásica es un síndrome caracterizado por un dolor facial paroxístico recurrente similar a una descarga eléctrica breve, de comienzo y final abrupto, generalmente unilateral, que afecta a una o más ramas del nervio trigémino y presenta periodos de remisión completa. Los pacientes refieren habitualmente zonas o estímulos desencadenantes (zonas trigger o puntos gatillos) como ciertos movimientos faciales (sonreír, masticar) o estímulos como el frío o calor.

Actualmente es aceptada como causa de la NT idiopática o primaria la compresión vascular del nervio trigémino en la zona de la entrada de la raíz (REZ) ${ }^{2,15}$. La primera línea de tratamiento es médico (carbamazepina, fenitoina, gabapentina). Sin embargo, ante el fracaso o intolerancia a

Abreviaturas. DMV: descompresión microvascular. NT: neuralgia del trigémino 
este, existen diferentes opciones de tratamiento como los métodos percutáneos, la radiocirugía y la descompresión microvascular (DMV).

En 1934 Dandy publicó en American Journal Surgery las primeras observaciones de la compresión vascular como etiología de la NT ${ }^{5}$. Gadner en 1966 fue el primero en realizar una $\mathrm{DMV}^{6} \mathrm{y}$ en las últimas décadas Jannetta ${ }^{7}$ ha popularizado esta técnica.

Actualmente es ampliamente aceptada como un método lógico y eficaz para el tratamiento de la NT típica en pacientes jóvenes y en ancianos con buenas condiciones clínicas 9 .

Presentamos nuestra experiencia con la DMV como tratamiento para la NT típica y evaluamos distintos factores de éxito o fracaso de la misma.

\section{Material y métodos}

Entre 1995 y 2005, 89 pacientes fueron intervenidos quirúrgicamente con DMV como tratamiento de la NT típica refractaria a tratamiento médico. Fueron excluidos del estudio las NT atípicas y las secundarias (esclerosis múltiple, tumores, MAV, etc.).

Presentamos las características demográficas y clínicas, los hallazgos quirúrgicos y la evolución postoperatoria. Para esto, clasificamos los resultados en tres categorías: 1) muy buena: cuando hay ausencia de dolor sin medicación; 2) buena: ante la presencia de la disminución parcial del dolor y/o de la medicación; y 3) mala: en los pacientes que continuaron con el mismo dolor y dosis de medicación preoperatoria.

Evaluamos los factores pronósticos en 86 pacientes al año posoperatorio, debido al fallecimiento de un paciente de causa no neurológica y a la pérdida de seguimiento en dos casos.

Los factores pronósticos estudiados fueron con respecto a: 1) el paciente (sexo, edad y antecedente de HTA); 2) la duración evolutiva de la neuralgia; 3) cantidad de ramas afectadas; 4) tipo de conflicto; 5) material utilizado para separar el conflicto neuro-vascular.

Todos los pacientes fueron operados en posición decúbito lateral con la técnica clásica. Se realizó una craniectomía retromastoidea y exploración del nervio trigémino con descompresión vascular del mismo. Esta separación fue realizada con músculo en 11 casos, Ivalon (esponja de polivinilo) en 25 y Gore-Tex en 50.

\section{Análisis estadístico}

Todas las variables fueron asumidas como categóricas describiéndose a través de proporciones. La diferencias entre grupos se evaluaron en su significación estadística a través de la prueba de $\chi^{2}$ fijando como valor de P menor de 0.05 .

\section{Resultados}

\section{Características demográficas y clínicas}

La edad media fue de 56 años con un rango de 32 a 71 años. 52 pacientes fueron hombres y 37 mujeres. El lado derecho fue el más afectado con un $68 \%$ de los casos.

La duración media de los síntomas fue de 2,3 años

\begin{tabular}{|c|c|}
\hline \multicolumn{2}{|c|}{$\begin{array}{c}\text { Tabla } 1 \\
\text { Características demográficas y clínicas }\end{array}$} \\
\hline Características & Valor \\
\hline \multicolumn{2}{|l|}{ Edad-años } \\
\hline Media & 56 \\
\hline Rango & $32-71$ \\
\hline Sexo masculino-n (\%) & $52(58)$ \\
\hline Lado derecho-n (\%) & $61(68)$ \\
\hline \multicolumn{2}{|c|}{ Evolución de la neuralgia-meses } \\
\hline Media & 27 \\
\hline Rango & $4-84$ \\
\hline \multicolumn{2}{|c|}{ Extensión de la neuragia-n (\%) } \\
\hline Una rama & $32(36)$ \\
\hline Dos ramas & $53(60)$ \\
\hline Tres ramas & $4(4)$ \\
\hline \multicolumn{2}{|c|}{ Distribución de la neuralgia-n (\%) } \\
\hline $\mathrm{V} 2$ & $19(21)$ \\
\hline $\mathrm{V} 3$ & $13(15)$ \\
\hline V1-V2 & $16(18)$ \\
\hline V2-V3 & $37(42)$ \\
\hline V1-V2-V3 & $4(4)$ \\
\hline
\end{tabular}

(rango: 4 meses a 7 años).

En 32 casos la distribución del dolor fue en una sola rama, V2 en 19 (21\%) y V3 en 13 (15\%); en 53 pacientes dos ramas, V2-V3 en $37(42 \%)$ y V1-V2 en $16(18 \%)$ y solamente $4(4 \%)$ pacientes presentaron compromiso de V1-V2-V3. (Tabla 1)

\section{Hallazgos quirúrgicos}

En todos los casos se evidenció una compresión vascular. Esta se produjo por una arteria en $73(82 \%)$ casos, una vena en $8(9 \%)$ casos y por una arteria y vena en $8(9 \%)$ casos. La compresión arterial fue causada por la arteria 
cerebelosa superior o algunas de sus ramas en 68 pacientes, la arteria cerebelosa anterioinferior o algunas de sus ramas en 4 pacientes y la arteria basilar en 1 paciente. La compresión del nervio fue en el tercio interno en 59 (81\%) casos y en el tercio medio en 14 (19\%) casos.

La compresión venosa fue producida por la vena petrosa superior y la vena pontina transversa. El conflicto se evidenció en el tercio externo del nervio en $6(75 \%)$ casos y en el tercio medio en $2(25 \%)$ casos.

\section{Complicaciones}

Las complicaciones que se presentaron en nuestra serie fueron: náuseas y vómitos transitorios en 34 pacientes; disminución de la audición y fístula de LCR en un caso cada uno; y meningitis aséptica en dos pacientes.

Tabla 2

Complicaciones posoperatorias

\begin{tabular}{|l|c|}
\hline \multicolumn{1}{|c|}{ Complicación } & n (\%) \\
\hline Nauseas y vómitos transitorios & $34(38)$ \\
\hline Fístula LCR & $1(1)$ \\
\hline Hipoacusia & $1(1)$ \\
\hline Meningitis aséptica & $2(2)$ \\
\hline Ataxia cerebelosa permanente & $1(1)$ \\
\hline
\end{tabular}

Un paciente presentó ataxia permanente por un infarto cerebeloso. (Tabla 2)

Ocho pacientes fueron reoperados por recurrencia del dolor. De estos, 6 casos eran por compresión arterial y habíamos utilizado músculo como material para separar el conflicto vasculo-nervioso. Intraoperatoriamente observamos un proceso fibroso y un engrosamiento de la aracnoides en la región del nervio, imposibilitando una correcta liberación. En estos casos realizamos una rizotomía selectiva, con una buena evolución con respecto al dolor pero con disestesias e hipoestesias faciales.

Los otros dos casos fueron por compresión venosa, y evidenciamos durante la cirugía una nueva compresión venosa en un caso y en el otro no encontramos conflicto neuro-vascular. Ambos pacientes mejoraron en el posoperatorio, pero presentaron nueva recurrencia a los 5 y 8 meses.

No se presentó mortalidad por la DMV en nuestra serie.

\section{Evolución posoperatoria}

La duración del seguimiento evolutivo fue de 1 mes a 8 años (media 4 años). Al mes del posoperatorio, de 89 pacientes tuvieron una muy buena evolución 75 (84\%), buena evolución $11(12 \%)$ y mala en $3(4 \%)$.

$\mathrm{Al}$ año de la cirugía, de 86 casos en seguimiento presentaron muy buena evolución 66 (77\%), buena evolución 15 (17\%) y mala en $5(6 \%)$.

A los cinco años del procedimiento 64 pacientes fueron controlados. De estos, 48 (75\%) presentaron muy buena evolución, $11(17 \%)$ buena evolución y en $5(8 \%)$ mala evolución. (Gráfico 1)

\section{Factores pronósticos}

Dentro de los factores evaluados no fueron estadísticamente significativos la edad y el antecedente de HTA.

Por el contrario, los siguientes factores fueron estadísticamente significativos: 1 ) sexo ( $\mathrm{p}=0.0418$ ): $86 \%$ de muy buena evolución en hombres y $64 \%$ en mujeres; 2 ) duración evolutiva de la neuralgia ( $\mathrm{p}=0.0155): 88 \%$ de muy buena evolución en pacientes de menos de 2 años del comienzo del dolor, $64 \%$ de 2 a 4 años y $66 \%$ en más de 4 años; 3 ) cantidad de ramas afectadas ( $\mathrm{p}=0.001$ ): $90 \%$ de muy buena evolución con compromiso de una rama, $80 \%$ con dos ramas y ningún caso con las tres ramas; 4) tipo de conflicto $(\mathrm{p}=0.001)$ : $88 \%$ de muy buena evolución con compresión arterial y $13 \%$ con venosa; y 5) material utilizado para la separación ( $\mathrm{p}=0.001): 86 \%$ de muy buena evolución con Gore-Tex, $80 \%$ con Ivalon y $27 \%$ con músculo.

\section{Discusión}

La NT es una enfermedad benigna que interfiere gravemente en la calidad de vida de los pacientes, y en algunas ocasiones, ha conducido al suicidio a los que la sufren.

La DMV es un tratamiento efectivo y no ablativo ampliamente utilizado mundialmente. La tasa de éxito inmediato varia entre $83-94 \% \%^{1-3,14,19,20,23,24}$ y la incidencia de recurrencia es del $10-46 \%^{1-3,14,19,21}$.

Algunos autores mostraron que repetidas DMV aumentan los riesgos de complicaciones ${ }^{4}$. Las recurrencias ocurren mayoritariamente durante los dos primeros años del posoperatorio $^{8,13}$. La tasa anual de recurrencia de la NT disminuye por debajo del $2 \%$ después de los cinco años postoperatorios y son menores del $1 \%$ tras diez años de la cirugía ${ }^{2}$.

Nuestros resultados de evolución posoperatorios a corto y mediano plazo son similares a los descritos en la bibliografía.

También coinciden los hallazgos intraoperatorios en cuanto a la localización de la compresión, tanto arterial (principalmente tercio proximal) como venosa (tercio 


\section{Gráfico 1}

Evolución posoperatoria

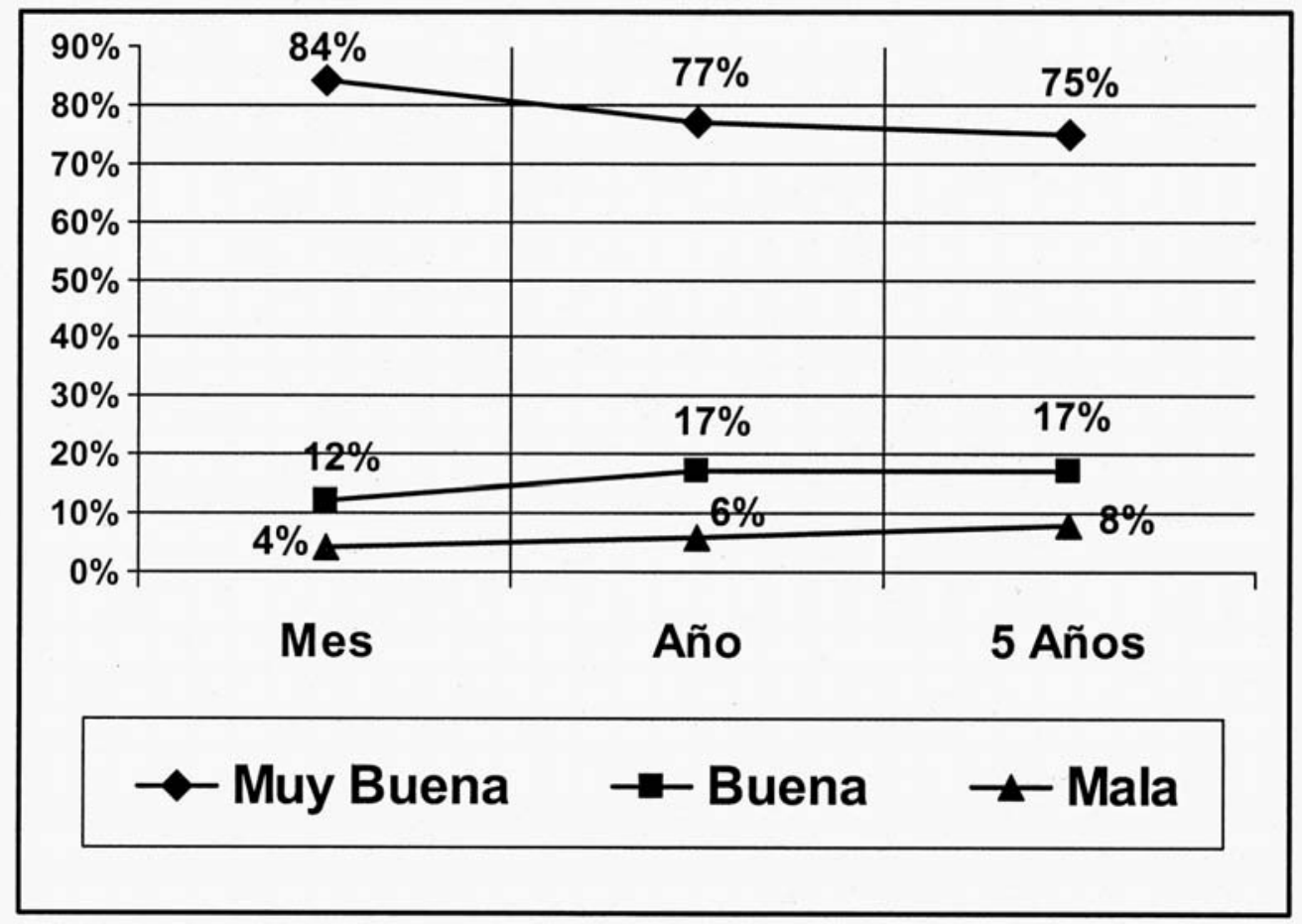

externo y medio) $)^{14,19}$

Las complicaciones de nuestra serie son comparables con las reportadas en la literatura ${ }^{2,14,19,23}$. No tuvimos mortalidad asociada a la DMV.

Están descritos como factores de excelente evolución a corto plazo la presencia de zonas trigger y el recuerdo del comienzo del dolor; y como desfavorables la realización de procedimientos percutáneos ablativos y/o DMV previos; y pérdida de la sensibilidad preoperatoria. A largo plazo son factores positivos de buena evolución también la presencia de zonas trigger ${ }^{24} \mathrm{y}$ negativo el dolor bilateral ${ }^{16,24}$.

Barker et $\mathrm{al}^{2}$ encontraron como factores predictores de alta tasa de recurrencia después de la DMV: el sexo femenino, la evolutividad de la sintomatología mayor de 8 años, la compresión venosa y la falta de mejoría posoperatoria inmediata.

Szapiro et $\mathrm{al}^{22}$ describió que el dolor paroxístico y el compromiso de una sola rama trigeminal se asocian a una excelente evolución. En su serie incluye un 35\% de pacientes que manifiestan dolor paroxístico y permanente en territorio trigeminal.

En nuestra serie fueron factores estadísticamente significativa de mala evolución al año posoperatorio: el sexo femenino, la duración evolutiva de la neuralgia mayor a 2 años, el compromiso de las tres ramas del trigémino, la compresión venosa y la utilización de músculo como material de separación entre el nervio y el elemento vascular.

Matsushima et al ${ }^{12}$ describieron como causa de la NT una compresión venosa en 25 pacientes $(20 \%)$ de 121 . Esta fue venosa pura en 7 casos $(5,7 \%)$, con una asociación al sexo femenino ( 6 casos), compromiso de 1 rama $(57,2 \%)$ y afección de la tercera rama $(71,5 \%)$. Seis de estos pacientes presentaron buena evolución posoperatoria con alivio del dolor.

En la serie de Sindou et a ${ }^{18}$, de 579 pacientes 155 $(27,6 \%)$ presentaron compresión venosa. Esta fue pura en $19(3,3 \%)$ y la desaparición del dolor a largo plazo fue del $66,7 \%$.

En nuestra serie la compresión venosa estuvo presente en 14 casos, siendo pura en 8 (9\%). Al año posoperatorio, solamente un paciente $(13 \%)$ tuvo muy buena evolución, tres buena evolución y cuatro mala. Reoperamos dos pacientes con buena evolución posoperatoria inmediata, pero presentaron una nueva recurrencia a los 5 y 8 meses.

La recurrencia por compresión venosa varia entre un 10 a $51 \%$ y se presenta principalmente durante el primer año posoperatorio $^{2,11,12,19}$.

La utilización de músculo (material reabsorbible) ha mostrado mayor capacidad de fibrosis y reabsorción de la prótesis con aumento de recurrencia del dolor ${ }^{10,16,26}$. Sza- 
piro et al $^{22}$ describió un peor pronóstico cuando se utilizó periostio en comparación con Dacron. Con prótesis de teflon se han descrito recidivas por formación de granulomas $^{25}$. Sindou et $a{ }^{18}$ ha demostrado una mejor evolución del dolor en los paciente que el material utilizado no tuvo contacto con el nervio y ha encontrado mejor evolución en 60 pacientes operados sin la utilización de material en comparación con el implante de teflon. También se ha utilizado al Spongostan con buenos resultados ${ }^{14}$.

\section{Conclusión}

Nosotros encontramos que la DMV es una técnica eficaz y segura para el tratamiento de la NT típica refractaria al tratamiento médico, con una baja tasa de morbi-mortalidad.

Asimismo, en nuestra serie encontramos que la afectación de las tres ramas trigeminales y las reintervenciones por recidiva dolorosa se asocian a un mal resultado terapéutico, debiéndose tener en cuenta en estos casos otra modalidad de tratamiento.

Consideramos que no se debe utilizar músculo como elemento de interposición vasculo-nerviosa debido a los malos resultados observados.

\section{Bibliografía}

1. Apfelbaum, R.I.: Surgery for tic douloureux. Clin Neurosurg 1983; 31: 351-368.

2. Barker, F.G. II, Jannetta, P.J., Bissonette, D.J., Larkins, M.V., Jho, H.D.: The long-term outcome of microvascular decompression for trigeminal neuralgia. N Engl J Med 1996; 334: 1077-1083.

3. Burchiel, K.J., Clarke, H., Haglund, M., Loeser, J.D.: Long-term efficacy of microvascular decompression in trigeminal neuralgia. J Neurosurg 1988; 69: 35-38.

4. Cho, D.Y., Chang, C.G., Wang, Y.C., Wang, F.H., Shen, C.C., Yang, D.Y.: Repeat operations in failed microvascular decompression for trigeminal neuralgia. Neurosurgery 1994; 35: 665-670.

5. Dandy, W.E.: Concerning the cause of trigeminal neuralgia. Am J Surg 1934; 24: 447-455.

6. Gardner, W.J., Miklos, M.V.: Response of trigeminal neuralgia to decompression of sensory root: "discussion of cause of trigeminal neuralgia". JAMA 1959; 170: 1773-1776.

7. Jannetta, P.J.: Arterial compression of the trigeminal nerve at the pons in patients with trigeminal neuralgia. J Neurosurg 1967; 26: 159-162.

8. Jannetta, P.J.: Surgical treatment: Microvascular decompression, in Fromm GH, Sessle BJ (eds): Trigeminal Neuralgia: Current Concepts Regarding Pathogenesis and Treatment. Boston, Butterworth-Heinemann, 1991, pp 145-158.

9. Keyoumars, A., Marsh, H.: Microvascular decompres- sion for trigeminal neuralgia in the elderly: a review of safety and efficacy. Neurosurgery 2004; 55: 840-850.

10. Klun, B.: Microvascular decompression and partial sensory rhizotomy in the treatment of trigeminal neuralgia: Personal experience with 220 patients. Neurosurgery 1992; 30: 49-52.

11. Lee, S.H., Levy, E.I., Scarrow, A.M., Kassam, A., Jannetta, P.J.: Recurrent trigeminal neuralgia attributable to veins after microvascular decompression. Neurosurgery 2000; 46: 356-361.

12. Matsushima, T., Huynh-Le, P., Miyazono, M.: Trigeminal neuralgia caused by venous compression. Neurosurgery 2004; 55: 334-339.

13. Mendoza, N., Illingworth, R.D.: Trigeminal neuralgia treated by microvascular decompression: A long-term followup study. Br J Neurosurg 1995; 9: 13-19.

14. Pamir, M.N., Peker, S.: Microvascular decompression for trigeminal neuralgia: a long-term follow-up study. Minim Invas Neurosurg 2006; 49: 342-346.

15. Pamir, M.N., Zirh, T.A., Ozer, A.F., Keles, G.E., Baykan, N.: Microvascular decompression in the surgical management of trigeminal neuralgia. Neurosurg Rev 1995; 18: 163-167.

16. Rath, S.A., Klein, H.J., Richter, H.P.: Findings and long-term results of subsequent operations after failed microvascular decompression for trigeminal neuralgia. Neurosurgery 1996; 39: 933-940.

17. Sindou, M., Amrani, F., Mertens, P.: Décompression vasculaire microchirurgicale pour néuralgie du trijumeau: Comparaison de deux modalités techniques et déductions physiopathiologiques-Étude sur 120 cas. Neurochirurgie 1990; 36: 16-26.

18. Sindou, M., Howeidy, T., Acevedo, G.: Anatomical observations during microvascular decompression for idiopathic trigeminal neuralgia. Prospective study in a series of 579 patients. Acta Neurochir (Wien) 2002; 144: 1-13.

19. Sindou, M., Leston, J., Howeidy, T., Decullier, E., Chapuis, F.: Micro-vascular decompression for primary trigeminal neuralgia (typical or atypical). Long-term effectiveness on pain; prospective study with survival analysis in a consecutive series of 362 patients. Acta Neurochir (Wien) 2006; 148: 1235-1245.

20. Slettebo, H., Eide, P.K.: A prospective study of microvascular decompression for trigeminal neuralgia. Acta Neurochir (Wien) 1997; 139: 421-425.

21. Sun, T., Saito, S., Nakai, O., Ando, T.: Long-term results of microvascular decompression for trigeminal neuralgia with reference to probability of recurrence. Acta Neurochir (Wien) 1994; 126: 144-148.

22. Szapiro, J.Jr., Sindou, M., Szapiro, J.: Prognostic factors in microvascular decompression for trigeminal neuralgia. Neurosurgery 1985; 17: 920-929.

23. Tronnier, V.M., Rasche, D., Hamer, J., Kienle, A.L., 
Kunze, S.: Treatment of idiopathic trigeminal neuralgia: comparison of long-term outcome after radiofrequency rhizotomy and microvascular decompression. Neurosurgery 2001; 48: 1261-1268.

24. Tyler-Kabara, E.C., Kassam, A.B., Horowitz, M.H., et al.: Predictors of outcome in surgically managed patients with typical and atypical trigeminal neuralgia: comparison of result following microvascular decompression. J Neurosurg 2002; 96: 527-531.

25. Vitali, A.M., Sayer, F.T., Honey, C.R.: Recurrent trigeminal neuralgia secondary to teflon felt. Acta Neurochir
(Wien) 2007; 149: 719-722.

26. Yamaki, T., Hashi, K., Niwa, J., et al.: Results of reoperation for failed microvascular decompression. Acta Neurochir (Wien) 1992; 115: 1-7.

Alberione, F.; Arena, A.; Matera, R.: Descompresiva microvascular en la neuralgia del trigémino: factores pronósticos. Neurocirugía 2008; 19: 242-247.

Correspondencia postal: Caseros 1857 (1152ABB) Capital Federal. Buenos Aires. Argentina. 